\title{
Is there any Association between Ventricular Ectopy and Falls in Community-Dwelling Older Adults?
}

\author{
Saman Parvaneh ${ }^{1,2,3}$, Bijan Najafi ${ }^{1,2,5}$, Nima Toosizadeh ${ }^{2}$, Irbaz Bin Riaz ${ }^{4}$, Jane Mohler ${ }^{1,2}$ \\ ${ }^{1}$ University of Arizona, Department of Surgery, Tucson, USA \\ ${ }^{2}$ University of Arizona, Department of Medicine, Arizona Center on Aging, Tucson, USA \\ ${ }^{3}$ Philips Research North America, Cambridge, USA \\ ${ }^{4}$ University of Arizona, Department of Internal Medicine, Tucson, USA \\ ${ }^{5}$ Baylor College of Medicine, Department of Surgery, Houston, USA
}

\begin{abstract}
Uni-channel ECG was recorded using a wearablesensor in 45 female elders aged 65 and above. Participants were categorized as non-fallers $(n=22)$ and fallers $(n=23)$ based on historical report of falling in the last 12 month. For data analysis, $P V C$ episodes were extracted using an automatic code developed in Matlab, and checked manually by an expert to ensure accuracy. Number of PVC episodes per hour was extracted as the parameter of interest in this study and compared between fallers and non-fallers using analysis of variance (ANOVA) test. Number of PVC episodes per hour was not significantly different $(p>0.05)$ between non-fallers (41.65 \pm 74.22$)$ and fallers (43.62 \pm 59.48$)$. In this study, the association of PVC frequency with fall risk and fear of fall was also studied; Number of PVC episodes were higher in high fall risk compared to low fall risk, and in high concern of fall compared to low concern of fall ( $p>0.05)$.
\end{abstract}

\section{Introduction}

Falls are common in older adults, one in three older adults age 65 years and older falls every year [1]. Over 800,000 patients are hospitalized annually after a fall [2], most often due to a head injury or hip fracture [3]. Furthermore, non-injurious falls can lead to fear of falling and spiraling deconditioning, adversely affecting quality of life and function [4].

Cardiovascular diseases (e.g., orthostatic hypotension, hypertension, and atrial fibrillation) are risk factors for falls [1]. Hence, assessment of ECG and cardiac autonomic nervous system is receiving more attention for risk of fall assessment [5-7].

Dizziness is one factor that is associated with higher risk of falling $[1,8]$. Frequent ventricular ectopic (premature ventricular contraction, PVC) episodes can cause dizziness due to the reduced ability of the heart to pump blood into systemic circulation $[9,10]$. The main goal of this study was to study the PVC frequency in community-dwelling older adults with, and without, history of falling.

\section{Method and material}

\subsection{Participants}

Forty-five female older adults without diagnosed cardiovascular disease participated in this study (age: $76.12 \pm 7.82$ years, BMI: $28.71 \pm 6.68 \mathrm{~kg} / \mathrm{m}^{2}$ ). Participants were recruited from geriatric clinics in a southwestern tertiary academic medical center, after completing informed consent approved by the University of Arizona Institutional Review Board.

\subsection{Fall, fall risk, and fear of falling assessment}

Participants were classified as non-faller and faller based on self-report history of fall in a prior 12 months, known as an established risk factor for future fall [11].

For assessment of fall risk, required time to complete a Timed Up and Go (TUG) test was used and participants with TUG time greater than 14 seconds were classified as high fall risk, and those 13 seconds and below categorized as low fall risk [12]. In this test, the participant rises from a chair, walks three meters, turns and returns to the chair, and sits [12, 13].

Validated questionnaire of 16-item fall efficacy scale (FES-I) was used for fear of fall assessment with the score ranges from 16 (no concern about falling) to 64 (severe concern about falling) [14]. Range 16-22 and 23-64 on FES-I score were used to group participants into low and high concern of falling, respectively [15]. 


\subsection{ECG analysis and PVC detection}

Four hour uni-channel ECG with sampling frequency of $250 \mathrm{~Hz}$ was recorded from participants using an FDAapproved chest-worn device, BioHarness3 (Zephyr Technology, Annapolis, MD). Of note, the ECG monitoring was done in participants' home, to represent their natural living environment. The algorithm for automatic detection of PVC episode(s) was developed in Matlab (Mathworks, Natick, MA). A Pan-Tompkins algorithm was employed for QRS detection and the detected $\mathrm{R}$ peaks were used for creating RR intervals time series [16]. Presence of a compensatory pause after PVC, a significant characteristic of PVC, was detected in RR intervals time series and was used for extraction of PVC episode(s) [10]. PVC episode(s) reviewed by an expert to ensure accuracy. Number of PVC episodes per hour (\#PVC/hour) was considered for ECG assessment.

\subsection{Statistical analysis}

The association between number of PVC(s) per hour and age was assessed using Pearson's correlation. Correlation range of 0.01-0.19, 0.20-0.39, 0.40-0.59, 0.600.79 , and $0.80-1.00$ were considered as very weak, weak, moderate, strong, and very strong association, respectively [17].

For evaluation of differences in demographics and health parameters among different groups (e.g., fallers vs. non-faller, low fall risk vs. high fall risk, and low fall concern vs. high fall concern), independent-samples t- test was used. One-way analysis of variance (ANOVA) test was used to evaluate the difference in number of PVC episodes per hour among different group, while considering age and body mass index (BMI) as covariates. A p-value of 0.05 was used as the critical statistical significance level.

\section{Results and discussion}

Every participants except one (97\%) had at least one PVC episode. Mean (standard deviation) of number of PVC per hour in all participants was 42.65 (66.32) (range: 0 -306). These results were in agreement with a previous study that reported at a least one PVC episode in $70 \%$ of elder participants [18]. Also, number of PVC episodes per hour were less than 100 in above mentioned study [18].

Number of PVC episodes per hour had a weak correlation with age $(r=0.28, p=0.076)$.

\section{1. $\quad$ PVC and falls}

Demographic and clinical characteristics of participants as well as number of PVC episodes per hour across nonfallers and fallers groups are listed in Table 1. No sociodemographic or baseline health information were significantly different between non-fallers and fallers $(p>0.05)$.

Using historical report of falling in the last 12 month, 22 participants were categorized as non-fallers (49\%) and 23 participants were classified as fallers (51\%). More specifically, thirteen had one fall, eight had two falls, one had four falls, and one had six falls.

Number of PVC episodes per hour was not significantly different ( $p=0.681$ ) between non-fallers $(41.65 \pm 74.22)$ and fallers (43.62 \pm 59.48$)$. When the number of PVCs was compared between non-fallers (41.65 \pm 74.22$)$ and fallers with just one fall (63.15 \pm 73.69$)$ an increasing trend in PVC frequency was observed $(p=0.857)$. Number of PVC episodes per hour versus number of fall(s) in the last 12 month is shown in Figure 1.

Table 1. Mean and standard deviation of demographic, health information, and number of PVC episodes per hour of participant across non-fallers and fallers groups (*: $p<$ $0.05)$.

\begin{tabular}{lccc}
\hline Characteristic & Non-faller & Faller & p-value \\
\hline Participants, $\mathrm{n}$ & 22 & 23 & - \\
Age, years & $75.34 \pm 7.89$ & $76.87 \pm 7.85$ & 0.518 \\
Height, cm & $158.62 \pm 7.53$ & $159.83 \pm 5.53$ & 0.547 \\
BMI, $\mathrm{kg} / \mathrm{m}^{2}$ & $29.82 \pm 6.77$ & $27.65 \pm 5.65$ & 0.281 \\
FES-I & $26.36 \pm 10.50$ & $27.91 \pm 12.58$ & 0.657 \\
TUG, s & $12.45 \pm 7.13$ & $14.31 \pm 8.55$ & 0.433 \\
\#PVC/hour & $41.65 \pm 74.22$ & $43.62 \pm 59.48$ & 0.681 \\
\hline BMI: Body Mass Index \\
FES-I: Fall efficacy scale international (16-64 scale) \\
TUG: Timed Up and Go time \\
\#PVC/hour: number of PVC episodes per hour \\
\hline
\end{tabular}

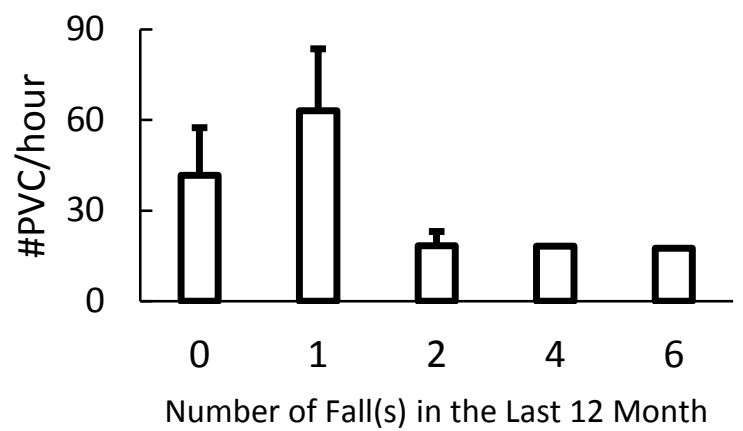

Figure 1. Number of PVC episodes per hour versus number of fall(s) in the last 12 month.

\subsection{PVC and fall risk}

Demographic, health information, and number of PVC episodes per hour of participant across low and high fall risk groups are listed in Table 2. Using time required to complete a TUG test, 31 and 14 participants were classified 
as low fall risk (69\%) and high fall risk (31\%), respectively. Age and fear of falling were significantly higher in high fall risk group compared to low fall risk group $(p<0.05)$.

Number of PVC episodes per hour was 55\% higher in high fall risk compared to low fall risk (number of PVC per hour: $36.46 \pm 58.29, P=0.709$ ).

Table 2. Mean and standard deviation of demographic, health information, and number of PVC episodes per hour of participant across low and high fall risk groups $(*$ : $\mathrm{p}<$ 0.05).

\begin{tabular}{lccc}
\hline Characteristic & $\begin{array}{c}\text { Low } \\
\text { Fall Risk }\end{array}$ & $\begin{array}{c}\text { High } \\
\text { Fall Risk }\end{array}$ & p-value \\
\hline Participants, n & 31 & 14 & - \\
Age, years & $73.55 \pm 6.01$ & $81.82 \pm 8.52$ & $0.001^{*}$ \\
Height, cm & $159.96 \pm 6.24$ & $157.65 \pm 7.12$ & 0.277 \\
BMI, kg/m ${ }^{2}$ & $28.09 \pm 6.32$ & $30.09 \pm 7.47$ & 0.359 \\
FES-I & $22.90 \pm 6.58$ & $36.57 \pm 14.48$ & $0.004^{*}$ \\
TUG, s & $9.52 \pm 2.14$ & $22.01 \pm 9.08$ & $<0.001^{*}$ \\
\#PVC/hour & $36.46 \pm 58.29$ & $56.37 \pm 82.18$ & 0.709 \\
\hline BMI: Body Mass Index & & \\
FES-I: Fall efficacy scale international (16-64 scale) & \\
TUG: Timed Up and Go time & & \\
\#PVC/hour: number of PVC episodes per hour & \\
\hline
\end{tabular}

\section{3. $\quad$ PVC and fear of falling}

Demographic, health information, and number of PVC episodes per hour for two group of low and high fall concern are listed in Table 3. Participants in high fall concern group were significantly older $(p=0.035)$ and slower in completing TUG test $(p=0.008)$ compared to the low fall concern group.

Although number of PVC per hour was $16 \%$ higher in the high fall concern group compared to the low fall concern (39.42 \pm 62.00$)$, the difference was non-significant $(p=0.728)$.

Although only PVC episodes were reported in this article, the results of this study have shown the potential for using a wearable sensor for monitoring heart arrhythmias, as well as PVC association with falling, fall risk, and fear of falling.

To reduce the effect of gender as a confounding parameter, only female participants were recruited in this study. Also, complex PVCs (e.g. multifocal and couplets) were not considered in this analysis. Future studies with larger sample size inclusive of both male and female participants are warranted to evaluate the association of heart arrhythmia with fall, fall risk, and fear of fall. Also, assessing dizziness and including continuous blood pressure measurement and cardiovascular measures (e.g., ejection fraction and cardiac output) in future studies can potentially improve the power of study for discussing the findings.
Table 3. Mean and standard deviation of demographic, health information, and number of PVC episodes per hour of participant across low concern and high concern of fall groups (*: $\mathrm{p}<0.05)$.

\begin{tabular}{lccc}
\hline Characteristic & $\begin{array}{c}\text { Low } \\
\text { Concern of } \\
\text { Fall }\end{array}$ & $\begin{array}{c}\text { High } \\
\text { Concern of } \\
\text { Fall }\end{array}$ & p-value \\
\hline Participants, n & 22 & 23 & - \\
Age, years & $73.63 \pm 4.80$ & $78.50 \pm 9.39$ & $0.035^{*}$ \\
Height, cm & $159.76 \pm 5.59$ & $158.74 \pm 7.42$ & 0.607 \\
BMI, kg/m ${ }^{2}$ & $26.75 \pm 6.81$ & $30.59 \pm 6.12$ & 0.053 \\
FES-I & $18.95 \pm 1.89$ & $35.00 \pm 11.40$ & $<0.001^{*}$ \\
TUG, s & $10.29 \pm 4.12$ & $16.37 \pm 9.39$ & $0.008^{*}$ \\
\#PVC/hour & $39.42 \pm 62.00$ & $45.75 \pm 71.46$ & 0.728 \\
\hline BMI: Body Mass Index & & \\
FES-I: Fall efficacy scale international (16-64 scale) & \\
TUG: Timed Up and Go time & & \\
\#PVC/hour: number of PVC episodes per hour &
\end{tabular}

\section{Conclusions}

In this study, associations between number of PVC per hour (ventricular ectopy frequency) and falls, fall risk, and fear of falling was studied. Number of PVC episodes were higher in fallers with one fall compared to non-fallers, in high fall risk compared to low fall risk, and in high fear of falling compared to low fear of falling. Future longitudinal studies are recommended to explore these associations in older adults with ventricular ectopy and different heart arrhythmia.

\section{Acknowledgements}

This study was partly supported by an STTR-Phase II Grant (award No. 2R42AG032748) from the National Institute on Aging and the Arizona Center on Aging's Hudson family. The content is solely the responsibility of the authors and does not necessarily represent the official views of the National Institute on Aging or the National Institutes of Health. We thank Marilyn Gilbert and the coordination team for helping with data collection and also Dr. Hossein Mehdikhani for assisting in manual review of PVC episodes.

\section{References}

[1] A. F. Ambrose, G. Paul, and J. M. Hausdorff, "Risk factors for falls among older adults: a review of the literature," Maturitas, vol. 75, pp. 51-61, 2013.

[2] C. f. D. Control and Prevention, "Web-based Injury Statistics Query and Reporting System (WISQARS) 2010," US Department of Health and Human Services, 2015.

[3] M. J. Goldacre, S. E. Roberts, and D. Yeates, "Mortality after admission to hospital with fractured neck of femur: database study," Bmj, vol. 325, pp. 868-869, 2002.

[4] R. G. Cumming, G. Salkeld, M. Thomas, and G. Szonyi, 
"Prospective study of the impact of fear of falling on activities of daily living, SF-36 scores, and nursing home admission," The Journals of Gerontology Series A: Biological Sciences and Medical Sciences, vol. 55, pp. M299-M305, 2000.

[5] R. Castaldo, P. Melillo, R. Izzo, N. De Luca, and L. Pecchia, "Fall prediction in hypertensive patients via short-term HRV Analysis," 2016.

[6] G. Grewal, S. Parvaneh, R. Menzies, T. Talal, D. Armstrong, M. Mohler, et al., "Heart rate response to measure fall-risk in patients with diabetes: a complementary measure," in Gerontologist, 2015, pp. 76-76.

[7] S. Parvaneh, G. Grewal, R.A. Menzies, T. K. Talal, D. G. Armstrong, J. Mohler, et al., "Heart rate response: a complementary measure for fall-risk assessment in patients with diabetic peripheral neuropathy," in 7th International Symposium on the Diabetic Foot, 2015.

[8] H. W. Lin and N. Bhattacharyya, "Impact of dizziness and obesity on the prevalence of falls and fall-related injuries," The Laryngoscope, vol. 124, pp. 2797-2801, 2014.

[9] D. J. Cantillon, "Evaluation and management of premature ventricular complexes," Cleveland Clinic journal of medicine, vol. 80, pp. 377-387, 2013.

[10] M. G. Tsipouras, D. I. Fotiadis, and D. Sideris, "An arrhythmia classification system based on the RR-interval signal," Artificial intelligence in medicine, vol. 33, pp. 237250, 2005.

[11] P. Stalenhoef, J. Diederiks, J. Knottnerus, A. Kester, and H. Crebolder, "A risk model for the prediction of recurrent falls in community-dwelling elderly: a prospective cohort study," Journal of clinical epidemiology, vol. 55, pp. 10881094, 2002.

[12] A. Shumway-Cook, S. Brauer, and M. Woollacott, "Predicting the probability for falls in community-dwelling older adults using the Timed Up \& Go Test," Physical therapy, vol. 80, pp. 896-903, 2000.
[13] D. Podsiadlo and S. Richardson, "The timed "Up \& Go": a test of basic functional mobility for frail elderly persons," Journal of the American geriatrics Society, vol. 39, pp. 142148, 1991.

[14] L. Yardley, N. Beyer, K. Hauer, G. Kempen, C. PiotZiegler, and C. Todd, "Development and initial validation of the Falls Efficacy Scale-International (FES-I)," Age and ageing, vol. 34, pp. 614-619, 2005.

[15] K. Delbaere, J. C. Close, A. S. Mikolaizak, P. S. Sachdev, H. Brodaty, and S. R. Lord, "The falls efficacy scale international (FES-I). A comprehensive longitudinal validation study," Age and ageing, vol. 39, pp. 210-216, 2010.

[16] J. Pan and W. J. Tompkins, "A real-time QRS detection algorithm," IEEE transactions on biomedical engineering, pp. 230-236, 1985.

[17] I. Lawrence and K. Lin, "A concordance correlation coefficient to evaluate reproducibility," Biometrics, pp. 255-268, 1989.

[18] K. A. Eagle and R. R. Baliga, Practical cardiology: evaluation and treatment of common cardiovascular disorders: Lippincott Williams \& Wilkins, 2008.

Address for correspondence.

Jane Mohler

Arizona Center on Aging, University of Arizona

1807 Elm Street, Tucson, AZ 85724

jmohler@aging.arizona.edu 